\title{
A Study of Semi-orthogonal Symmetric Multiple Vector-valued Wavelets with poly-scale and Applications
}

\author{
Deyou Yuan \\ School of Education, Nanyang Institute of Technology, Nanyang 473000, China \\ zas123qwe@126.com
}

\begin{abstract}
Keywords: multiple vector-valued wavelets, symmetric vector-valued scaling functions, subdivision operators, finite support, symmetric wavelets, polyphase matrix

Abstract. Material science is an interdisciplinary field applying the properties of matter to various areas of science and engineering. In this work, we introduce orthogonal vector-valued wavelets with poly-scale, which are wavelets for vector fields, based on the notion of full rank subdivision oper -ators. It is demonstrated that, like in the scalar and multiwavelet case, the existence of an symmetr -ic vector-valued scaling function guarantees the existence of symmetric vector-valued wavelet func -tions. Secondly, we propose a construction algorithm for compactly supported orthogonal twodirectional matrix-valued wavelet packets. Lastly, A method for constructing a class of compactly supported orthogonal matrix-valued wavelets is presented by means of matrix theory.
\end{abstract}

\section{Introduction}

Mechanical engineering science emerged in the 19th century as a result of developments in the field of physics. Mechanical engineering overlaps with aerospace engineering, metallurgical engineering, civil engineering, electrical engineering, petroleum engineering, manufacturing engine -ering, chemical engineering, and other engineering disciplines to varying amounts. Since the late 20th century, multiwavelet theory have become the focus in the wavelet analysis. Multiwavelets, which can offer properties like symmetry, orthogonality, short support at the same time, have been widely used in signal procssing, image processing and so on. Orthogonal two-direction multiple vector-valued wavelets with multi-scale is researched. The concept of two-direction vector-valued multiresolution analysis is introduced. The two-direction vector-valued wavelets and wavelet pack -ets with multi-scale are presented. We present a construction algorithm of compactly supported orthogonal two-direction vector-valued wavelets by means of the matrix theory and the timefrequency analysis method. Properties of a class of orthogonal two-direction vector-valued wavelet packets are characterized.Vector-valued wavelet is a class of more generalized wavelets. The notion of vector-valued wavelets is introduced. The existence and the method for construction of the orthogonal vector-valued wavelets are studied in [1] by Xia and Suter. One of the basic advantages of wavelets is that an event can be simultaneously described in the fre-quency domain as well as in the time domain, unlike the usual Fourier transform where an e vent is accurately described either in the frequency or in the time domain. Fowler and Li using discrete orthogonal vector-valued wavelet transform to study Marine eddy current phenomenon. To obtain some beautiful features, Yang S Z [2] introduce the concepts of two-direction scaling function and two-direction wavelets, and give the definition of orthogonal two-direction scaling function and construct the orthogonal twodirection wavelets. Compared with general wavelet, two-direction wavelets filter can be flexible to choose in practical application [3]. Naturally, two-direction wavelets has become a key research 
object. Based on an observation in Chen [4] and using blocking partition and full rank subdivision operator developed by Micchelli and Sauer [5] and some ideas from Bacchelli [6], one of the pur pose of this paper is to extend the result in [6] to the case of multi-scale orthogonal matrix-valued wavelets. In the meantime we propose an algorithm for constructing a class of orthogonal compa -ctly supported matrix-valued wavelets with multi-scale.

\section{On multiple vector-valued multiresolution analysis}

By $L^{2}\left(R, C^{s}\right):=\left\{\hbar(t):=\left(h_{1}(t), h_{2}(t), \cdots, h_{s}(t)\right)^{T}, h_{l}(t) \in L^{2}(R), l=1,2, \cdots, s\right\}$, we denote the space of square integrable vector-valued function, where $T$ is the transpose, where $s, r, m \in Z_{+}$be constants and $2 \leq s \leq+\infty, 2 \leq m \leq+\infty$. The space of all matrix-valued functions $L^{2}\left(R, C^{s \times r}\right)$ is defined as

$$
\begin{aligned}
& L^{2}\left(R, C^{s \times r}\right):=\left\{\mathrm{G}(t)=\left(\begin{array}{cccc}
g_{11}(t) & g_{12}(t) & \cdots & g_{1 r}(t) \\
g_{21}(t) & g_{22}(t) & \cdots & g_{2 r}(t) \\
\cdots & \cdots & \cdots & \cdots \\
g_{s 1}(t) & g_{s 1}(t) & \cdots & g_{s r}(t)
\end{array}\right): \begin{array}{c}
g_{i j}(t) \in L^{2}(R) \\
j=1,2, \cdots, s \\
j=1, \cdots, r
\end{array}\right\} \\
& \iiint_{\omega \in \mathrm{K}^{3}} \Phi(\eta) d \eta=\sum_{k \in Z^{3}} \iiint_{\omega \in \mathrm{K}^{3}}|\hat{\phi}((\eta+k) / b)|^{2} d \eta=\iiint_{\omega \in R^{3}}|\hat{\phi}(\eta / b)|^{2} d \eta<+\infty \\
& \langle\boldsymbol{S} \phi, g\rangle=\left\langle\sum_{v \in \Gamma}\left\langle\phi, h_{v}\right\rangle h_{v}, g\right\rangle=\sum_{v \in \Gamma}\left\langle\phi, h_{v}\right\rangle\left\langle h_{v}, g\right\rangle \\
& \left.=\sum_{v \in \Gamma} \overline{\left\langle g, h_{v}\right\rangle}\left\langle\phi, h_{v}\right\rangle=<\phi, \sum_{v \in \Gamma}\left\langle g, h_{v}\right\rangle h_{v}\right\rangle=\langle\phi, \boldsymbol{S} g\rangle, \quad\left\langle\boldsymbol{S}^{-1} \phi, g\right\rangle=\left\langle\boldsymbol{S} \boldsymbol{S}^{-1} \phi, \boldsymbol{S}^{-1} g\right\rangle=\left\langle\phi, \boldsymbol{S}^{-1} g\right\rangle
\end{aligned}
$$

where $\mathrm{T}$ means the transpose of a vector or a matrix, and $n \in \mathrm{Z}$ is a constant as well as $n \geq 2$. By $I_{s}$, we denote the identity matrix. For arbitrary $\hbar(t) \in L^{2}\left(R, C^{s}\right)$, its integration is defined to be

$$
\int_{R} \hbar(t) d t:=\left(\int_{R} h_{1}(t) d t, \int_{R} h_{2}(t) d t, \cdots, \int_{R} h_{s}(t) d t\right)^{T}
$$

For any vector-valued function $\hbar(t) \in L^{2}\left(R, C^{s}\right)$, its Fourier transform is defined as follows

$$
\hat{\hbar}(\eta):=\int_{R} \hbar(t) \exp \{-i \eta t\} d t, \eta \in R .
$$

For arbitrary vector-valued function $\hbar \in L^{2}\left(R, C^{s}\right),\|\hbar\|_{2}$ denotes the norm of $\hbar(t)$ as

$$
\|\hbar\|_{2}:=\left(\sum_{t=1}^{s} \int_{R}\left|h_{t}(t)\right|^{2} d t\right)^{1 / 2},
$$

The norm, Fourier transform and the integration of $\Psi(t) \in L^{2}\left(R, C^{s \times r}\right)$ is defined similarly as a single vector-valued function $\hbar(t)$. For arbitrary $\hbar(t) \in L^{2}\left(R, C^{s}\right), \operatorname{set} \hbar_{t: v, n}=m^{v / 2} h_{t}\left(m^{v} t-n\right) \imath=1,2$, $\cdots, s$, For $\mathbf{G}(t) \in L^{2}\left(R, C^{s \times r}\right), \boldsymbol{\Phi}(t) \in L^{2}\left(R, C^{s \times u}\right)$, their symbol inner product is defined by

$$
\langle\mathbf{G}, \mathbf{\Phi}\rangle:=\int_{R} \mathbf{G}(t) * \mathbf{\Phi}(t) d t,
$$

where $\mathbf{G}(t) *$ means the transpose and the complex conjugate of $\mathbf{G}(t)$.

Definition 1. We say that a matrix-valued function $\mathrm{G}(t) \in L^{2}\left(R, C^{s \times s}\right)$ is an orthogonal one, if it has orthonormal integer translates, which means that

$$
\langle\mathrm{G}(\cdot), \mathrm{G}(\cdot-n)\rangle=\delta_{0, n} I_{s}, \quad n \in Z
$$

where $I_{s}$ denotes the $s \times s$ identity matrix and $\delta_{0, n}$ is the Kronecker symbol, i.e., for the case of $n=0, \delta_{0, n}=1$; otherwise, $\delta_{0, n}=0$. Similarly, if a vector-valued functions $\Lambda(t) \in L^{2}\left(R, C^{s}\right)$, satisfy $\langle\Lambda(t), \Lambda(t-n)\rangle=\delta_{0, n}$, then it is called an orthogonal vector-valued function. If two vector-valued functions $\Psi(t), \Lambda(t) \in L^{2}\left(R, C^{s}\right)$ satisfy $\langle\Psi(t), \Lambda(t-n)\rangle=\delta_{0, n}$, then they are called mutually biorth 
-ogonal vector-valued functions. We say that two matrix -valued functions $\boldsymbol{\Psi}(t), \boldsymbol{\Phi}(t) \in L^{2}\left(R, C^{s \times s}\right)$ are mutually biorthogonal, if they satisfy

$$
\langle\boldsymbol{\Psi}(\cdot), \boldsymbol{\Phi}(\cdot-n)\rangle=\delta_{0, n} I_{s}, n \in Z .
$$

Lemma 1. A matrix-valued function $\Psi(t)=\left(\Psi_{1}(t), \Psi_{2}(t), \cdots, \Psi_{s}(t)\right)$ is an orthogonal one if and only if its integer translates of the column vectors $\Psi_{t}(t), \imath=1,2, \cdots, s$ are mutually biorthogonal.

Let $H(t)=\left(\hbar_{1}(t), \hbar_{2}(t), \cdots, \hbar_{s}(t)\right)$ satisfy the following matrix refinement equation,

$$
H(t)=\sum_{n \in Z} P(n) H(m t-n),
$$

where $\underline{P}=\left(P(n) \in C^{s \times s}: n \in Z\right) \in \ell_{00}(Z)^{s \times s}$ is a finitely supported matrix mask, which has only a finite non-zero terms, and $\hbar_{l}(t) \in L^{2}\left(R, C^{s}\right), \imath=1,2, \cdots, s$, are the column vectors of $H(t)$.

Lemma 2. Suppose that $H(t)$, defined in (4), is an orthogonal multiple vector-valued scaling function. Then, for any $\sigma \in Z$, we have

$$
\sum_{n \in Z}(P(n+m \sigma)) * P(n)=m \delta_{0, \sigma} I_{s}, \quad 2 \leq m \leq+\infty .
$$

Define a sequence of closed subspaces $U_{j} \subset L^{2}\left(R, C^{s \times s}\right)$ by

$$
U_{j}=\left\{H(t): H(t)=\sum_{n \in Z} P(n) H(m t-n), P(n) \in \ell_{00}(Z)^{s \times s} .\right.
$$

Definition 2. We say that $H(t)$ in (4) yields a matrix-valued multiresolution analysis $\left\{U_{j}\right\}_{j \in Z}$ of $L^{2}\left(R, C^{s \times s}\right)$, if the sequence $\left\{U_{j}\right\}_{j \in Z}$, defined in (6) meets the following conditions, (i) $U_{j} \subset U_{j+1}$, $\forall j \in Z$ (ii) $\bigcap_{j \in Z} U_{j}=\{0\} ; \bigcup_{j \in Z} U_{j}$ is dense in $L^{2}\left(R, C^{s \times s}\right)$; (iii) $\boldsymbol{\Psi}(\cdot) \in U_{0} \Leftrightarrow \boldsymbol{\Psi}\left(m^{j} \cdot\right) \in U_{j}, j \in Z$; (iv) there exist $H(t) \in U_{0}$ such that $\{H(t-n)\}_{n \in Z}$ form a Riesz basis of the subspace $U_{j}$.

Lemma 3. Suppose that $F_{r}(t), r=1,2, \cdots, m-1$ defined in (7), are a pair of mutually biorthogonal matrix-valued wavelet functions associated with an orthogonal matrix-valued scaling function $H(t)$. Then for any integer $\sigma \in Z$, we have

$$
\begin{gathered}
\sum_{n \in Z}(P(n+m \sigma)) * B_{r}(n)=O, \quad r=1,2, \cdots m-1, \quad 2 \leq m \leq+\infty, \\
\sum_{n \in Z}\left(B_{r}(n+m \sigma)\right) * B_{l}(n)=m \delta_{r, l} \delta_{0, \sigma} I_{s}, \quad r, l=1,2 \cdots, m-1,2 \leq m \leq+\infty .
\end{gathered}
$$

In the following, two-direction refinable function is introduced. $\Phi(t)$ is called two-direction vec tor-valued refinable function, if $\Phi(t) \in L^{2}\left(R, C^{s}\right)$ satisfies the below two-direction refinable equation

$$
\Phi(t)=\sum_{k \in Z} p_{k}^{+} \Phi(m t-k)+\sum_{k \in Z} p_{k}^{-} \Phi(k-m t),
$$

where $a \in Z_{+}, a \geq 2$ and the finitely supported sequences of $n \times n$ matrices $\left\{p_{k}^{+}\right\}_{k \in Z},\left\{p_{k}^{-}\right\}_{k \in Z}$ are called positive-direction mask and negative-direction mask, respectively.

Definition $3^{[4]}$. We say that the vector-valued function $\Phi(t) \in L^{2}\left(R, C^{s}\right)$ is orthogonal two-dir -ection vector-valued function, if it satisfies

$$
\langle\Phi(t), \Phi(t-k)\rangle=\delta_{0, k} I_{s}, \quad\langle\Phi(t), \Phi(k-t)\rangle=0, k \in Z .
$$

A two-direction wavelets $\Psi_{\alpha}(x)$ associated with a two-direction vector-valued refinable function $\Phi(x)$ must satisfies the following equation

$$
\Psi_{\alpha}(t)=\sum_{k \in Z} q_{k}^{(\alpha)+} \Phi(a t-k)+\sum_{k \in Z} q_{k}^{(\alpha)-} \Phi(k-a t), \quad \alpha \in \Lambda .
$$

Definition 4. Let $\Phi(t)$ be an orthogonal two-direction vector-valued scaling function, and $\Psi_{\alpha}(t)$ satisfy the Equation (5), where $\alpha \in \Lambda$. If $\Phi(x)$ and $\Psi_{\alpha}(x)$ satisfy the following conditions, 


$$
\left\{\begin{array}{l}
<\Phi(t), \Psi_{\alpha}(t-k)>=0, \quad<\Phi(t), \Psi_{\alpha}(k-t)>=0, \\
<\Psi_{\alpha}(t), \Psi_{\beta}(t-k)>=\delta_{\alpha, \beta} \delta_{0, k},<\Psi_{\alpha}(t), \Psi_{\beta}(k-t)>=0 .
\end{array}\right.
$$

Then, $\Psi_{\alpha}(x)$ are called orthogonal two-direction vector-valued wavelet functions with $\Phi(x)$.

Theorem $1^{[6]}$ If $\Phi(t)$ is an orthogonal two-direction vector-valued scaling functions, then we have

$$
\left\{\begin{array}{l}
\sum_{k \in z}\left[p_{k}^{+}\left(p_{k+a l}^{+}\right)^{*}+p_{k}^{-}\left(p_{k+a l}^{-}\right)^{*}\right]=a \delta_{0, l} I_{s} \\
\sum_{k \in z}\left[p_{k}^{+}\left(p_{k+a l}^{-}\right)^{*}+p_{k}^{-}\left(p_{k+a l}^{+}\right)^{*}\right]=0
\end{array}, l \in Z, k \in Z .\right.
$$

\section{The charateristics of a class of marix-valued wavelet wraps}

Let $G_{0}(t)=H(t), G_{\mu}(t)=\Psi_{\mu}(t), M_{k}^{(0)}=P_{k}, M_{k}^{(\mu)}=B_{k}^{(\mu)}, \mu \in \Lambda, k \in Z, \mathrm{r}=1$. For any $\beta \in Z_{+}$and the given matrix-valued orthogonal scaling functions $G_{\underline{0}}(t)$, iteratively define, as follows

$$
G_{\beta}(t)=G_{a \sigma+\mu}(t)=\sum_{k \in Z} M_{k}^{(\mu)} G_{\sigma}(m t-k),
$$

where $\mu \in \Lambda_{0}=\Lambda \bigcup\{0\}, \sigma \in Z_{+}$is the unique element such that $\beta=a \sigma+\mu, \mu \in \Lambda_{0}$ follows.

Definition 5. We say that one family $\left\{G_{a \sigma+\mu}(t), \sigma \in Z_{+}, \mu \in \Lambda_{0}\right\}$ is matrix-valued wavelet wraps with respect to the orthogonal matrix-valued scaling function $G_{0}(t)$, where $G_{a \sigma+\mu}(t)$ are given by (9).

Proposition $2^{[7]}$. Assume that $\left.\left\{G_{\beta} t\right), \beta \in Z_{+}\right\}$is vector-valued wavelet wraps with respect to the orth -ogonal vector-valued functions $G_{0}(t)$. Then, for $\beta \in Z_{+}, \mu, v \in \Lambda_{0}$, we have

$$
\left\langle G_{a \beta+\mu}(\cdot), G_{a \beta+v}(\cdot-k)\right\rangle=\delta_{0, k} \delta_{\mu, v} I_{s}, k \in Z .
$$

\section{Conclusion}

We introduce orthogonal matrix-valued wavelets with poly-scale, which are wavelets for vector fie -lds. The properties of two-directional matrix-valued wavelet wraps are characterized.

\section{Acknowledgement}

This work was supported by the Science Research Foundation of Education Department of Shaanxi Provincial Government (Grant No: 2013JK0564, \& 12JK0882,\& 12JK1107).

\section{References}

[1] X. Xia, B W Suter. Vector-Valued wavelets and vector filter banks. IEEE Trans Signal Pro -cessing, 1996, 44(3): 508-518.

[2] S.Yang, Y.Li. Two-direction refinable functiongs and two- direction wavelets with dilation factor m. Applied Mathematics and Computation, 2007, 188 (2): 1908-1920.

[3] N. Zhang, X.Wu X. Lossless Compression of Color Mosaic Images. IEEE Trans Image processing, 2006, 15(16): 1379-1388.

[4] Q. Chen, A. Huo. The research of a class of biorthogonal compactly supported vector-valued wavelets. Chaos, Solitons \& Fractals, 2009,41(2): 951-961.

[5] S. Bacchelli, et al. Wavelets for multichannel signals. Adv Appl Math. 2002,29(4): 581-598.

[6] Q. Chen, Y. Zhao, H. Gao. Existence and characterization of orthogonal multiple vector- valued wavelets with three-scale. Chaos, Solitons \& Fractals. 2009,42(4): 2484-2493. 